ARTICLE

\title{
RNA-mediated control of cell shape modulates antibiotic resistance in Vibrio cholerae
}

Nikolai Peschek (1) 1,2,7, Roman Herzog 1,2,7, Praveen K. Singh (10 3,7, Marcel Sprenger ${ }^{1,7}$, Fabian Meyer ${ }^{2,4}$,

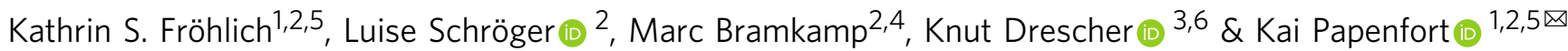

Vibrio cholerae, the cause of cholera disease, exhibits a characteristic curved rod morphology, which promotes infectivity and motility in dense hydrogels. Periplasmic protein CrvA determines cell curvature in $V$. cholerae, yet the regulatory factors controlling CrvA are unknown. Here, we discover the VadR small RNA (sRNA) as a post-transcriptional inhibitor of the crvA mRNA. Mutation of vadR increases cell curvature, whereas overexpression has the inverse effect. We show that vadR transcription is activated by the $V x r A B$ two-component system and triggered by cell-wall-targeting antibiotics. $V$. cholerae cells failing to repress crvA by $V$ adR display decreased survival upon challenge with penicillin $G$ indicating that cell shape maintenance by the sRNA is critical for antibiotic resistance. VadR also blocks the expression of various key biofilm genes and thereby inhibits biofilm formation in V. cholerae. Thus, VadR is an important regulator for synchronizing peptidoglycan integrity, cell shape, and biofilm formation in $V$. cholerae.

\footnotetext{
${ }^{1}$ Institute of Microbiology, Friedrich Schiller University, 07745 Jena, Germany. ${ }^{2}$ Faculty of Biology, Ludwig-Maximilians-University of Munich, 82152 Martinsried, Germany. ${ }^{3}$ Max Planck Institute for Terrestrial Microbiology, 35043 Marburg, Germany. ${ }^{4}$ Institute for General Microbiology, ChristianAlbrechts-University, Kiel, Germany. ${ }^{5}$ Microverse Cluster, Friedrich Schiller University Jena, 07743 Jena, Germany. ${ }^{6}$ Department of Physics, PhilippsUniversität Marburg, 35032 Marburg, Germany. ${ }^{7}$ These authors contributed equally: Nikolai Peschek, Roman Herzog, Praveen K. Singh, Marcel Sprenger.

凶email: kai.papenfort@uni-jena.de
} 
B acterial cell shape is highly diverse and tightly conserved at the species level. Certain cell morphologies have been associated with distinct physiological functions such as optimized nutrient uptake, efficient surface adherence, and increased evasion from protist grazing ${ }^{1}$. Cell shape is determined by the geometry of the cell-wall, which can be affected by filamentous proteins that change or interfere with peptidoglycan insertion ${ }^{2-4}$. For example, the cytoskeleton-like filament, crescentin (CreS), controls cell curvature in the model bacterium Caulobacter crescentus $^{5}$. In Vibrio cholerae, CrvA protein polymerizes in the periplasmic space to promote cell bending $6,7 . \mathrm{V}$. cholerae cells lacking the $\operatorname{crvA}$ gene display attenuated colonization in animal infection models and it has been reported that cell curvature of $V$. cholerae increases in a cell-density dependent manner6. These findings indicate that CrvA levels are continuously adjusted during growth, however, the necessary regulatory factors are currently unknown.

Recently, post-transcriptional control by small regulatory RNAs (sRNAs) in V. cholerae was shown to be key for modulating spatiotemporal processes such as virulence, biofilm formation, secondary messenger production, and stress resistance ${ }^{8-11}$. The largest class of sRNAs associates with the RNA chaperone Hfq and typically regulates the expression of target mRNAs by base-pairing via short stretches of imperfect complementarity ${ }^{12,13}$. The network regulated by a single sRNA frequently involves dozens of targets and therefore sRNAs can rival transcription factors with respect to their regulatory scope and biological importance ${ }^{14}$. For example sRNAs are crucial for iron, membrane, and sugar homeostasis, as well as motility, biofilm formation, and virulence ${ }^{15,16}$, however, no sRNA has been yet reported to control cell shape.

Here, we employed the curved rod-shaped bacterium $V$. cholerae as a model system to study the impact of sRNAs on cell curvature. We discovered that production of the VadR ( VxrB activated small RNA, see below) sRNA efficiently reduced cell curvature in $V$. cholerae by inhibiting the expression of the $\operatorname{crv} A B$ mRNA. VadR expression is controlled by the VxrAB twocomponent system (a.k.a. WigKR ${ }^{17,18}$ ) and is activated by Blactam antibiotics. $V$. cholerae mutants deleted for $v a d R$ display increased sensitivity towards penicillin and we pinpoint this phenotype to VadR-mediated repression of $\operatorname{cr} v A B$. In addition, VadR controls several key genes required for biofilm assembly, including the mRNA encoding the RbmA matrix protein ${ }^{19}$. Thus, VadR also inhibits biofilm formation in $V$. cholerae. Our results reveal how a non-coding RNA regulates a cytoskeleton-like filament in bacteria, and establish a link between cell shape, biofilm formation, and antibiotic resistance in $V$. cholerae.

\section{Results}

VadR is an Hfq-dependent sRNA affecting cell curvature in $\boldsymbol{V}$. cholerae. To identify sRNAs regulating cell curvature in $V$. cholerae, we performed a microscopy-based forward genetic screen. We selected 21 uncharacterized sRNAs candidates from a pool of recently identified Hfq-dependent sRNAs $^{20}$ and cloned their respective genes onto multicopy plasmids. We transferred these plasmids into $V$. cholerae and assayed the resulting strains for centerline curvature using phase contrast microscopy. In line with a previous report ${ }^{6}$, we found that curvature decreased $\sim 3$-fold in crvA deficient cells, when compared to wild-type $V$. cholerae (Fig. 1a). Overexpression of 20 sRNAs did not render curvature significantly, however, cells overexpressing one sRNA, which we term VadR (a.k.a. Vcr09020, see below), displayed $\sim 2$-fold reduced curvature (Fig. 1a).

The $\operatorname{vadR}$ gene is located on the plus strand of the smaller $V$. cholerae chromosome between the vca0002 and vca0003 genes ${ }^{20}$.
The sRNA is present in numerous other Vibrios and carries a highly conserved $5^{\prime}$ end (Fig. 1b) frequently involved in RNA duplex formation with trans-encoded target mRNAs ${ }^{8,21}$. Structure probing experiments confirmed that this region is unstructured and therefore available for base-pairing with other transcripts (Supplementary Fig. 1a, b). Northern blot analysis revealed that VadR accumulates as a $\sim 85 \mathrm{nt}$ transcript and is most highly expressed at low cell densities (Fig. 1c). Stability of VadR was $\sim 3$ min in $V$. cholerae wild-type cells and $\sim 4$-fold reduced in cells lacking the $h f q$ gene (Supplementary Fig. 1c). Together, we conclude that VadR is a Hfq-dependent sRNA that is likely to act by base-pairing with other transcripts.

The VxrAB system activates VadR expression. Alignment of $\operatorname{vadR}$ promoter sequences revealed three conserved elements upstream the -10 box (Fig. 1b). While we were unable to directly assign a transcriptional regulator to these elements, we discovered that a $v a d R$ transcriptional reporter was $\sim 150$-fold more active in $V$. cholerae when compared to Escherichia coli (Supplementary Fig. 1d). These results suggested that vadR expression depended on a $V$. cholerae-specific factor, which allowed us to perform another genetic screen. Here, we employed a plasmid library expressing $\sim 2.5 \mathrm{~kb} V$. cholerae genomic fragments, which we cotransformed with a PvadR::lacZ transcriptional reporter into $E$. coli. We assayed $\sim 23,000$ colonies for $ß$-galactosidase activity on plates containing X-gal and isolated seven blue colonies. Sequence analysis of the respective plasmids revealed that all mapped to the vxrABCDE (vca0565-0569) locus; five plasmids contained sequences of $\operatorname{vxr} A B$ and two plasmids contained sequences of vxrABCDE (Supplementary Fig. 1e). To corroborate these results, we monitored $v a d R$ production in wild-type and $\triangle v x r A B C D E V$. cholerae by means of (i) promoter activity measurements and (ii) Northern blot analysis. Indeed, promoter activity was $\sim 50$-fold reduced in the $\operatorname{vx} A B C D E$ mutant (Supplementary Fig. 1f) and VadR was no longer detectable on Northern blots (Fig. 1d). Successive complementation of the $\operatorname{vx} A B C D E$ genes from a plasmid revealed that $\operatorname{vxr} A B$ (encoding the histidine kinase and response regulator of the two-component system, respectively) restored VadR expression, while vxrCDE were dispensable for regulation (Fig. 1d). Finally, to pinpoint direct regulation of $\mathrm{vadR}$ by VxrB, we reanalyzed previously reported ChIP-Seq data ${ }^{22}$ for binding of $\mathrm{VxrB}$ at the $\operatorname{vadR}$ promoter. Indeed, we discovered a pronounced, VxrB-specific peak upstream of the $v a d R$ gene (Supplementary Fig. 1g). These analyses also revealed a putative VxrB binding motif (TTGACAAAA-N2-TTGAC), which matched the three conserved sequence elements in the $\operatorname{vadR}$ promoter (Fig. 1b). Deletion of each of these sites efficiently reduced $v a d R$ promoter activity with sites 2 and 3 being most critical for transcription activation (Supplementary Fig. $1 \mathrm{~h}$ ). We also tested the effect of deleting the spacer sequence between sites 1 and 2, as well as between sites 2 and 3 on the $v a d R$ promoter. In both cases, we observed a strong drop in activity (Supplementary Fig. 1h). Together, we conclude that VadR is a VxrAB-activated sRNA that might be involved in regulation of cell shape in $V$. cholerae.

VadR regulates multiple genes involved in biofilm formation. To explore the molecular mechanism of VadR-mediated inhibition of cell bending, we next aimed to identify base-pairing partners of VadR in vivo. We used RNA-seq analyses to assess changes in global transcriptome levels following transient (15 min) overexpression of $v a d R$ in a $\Delta v a d R V$. cholerae strain. In total, 28 mRNAs, including $c r v A$, displayed significant changes following VadR expression (Fig. 2a and Supplementary Table 1). We validated regulation of all targets, except $i b p A$, using quantitative real-time PCR (by testing all monocistronic genes and the 

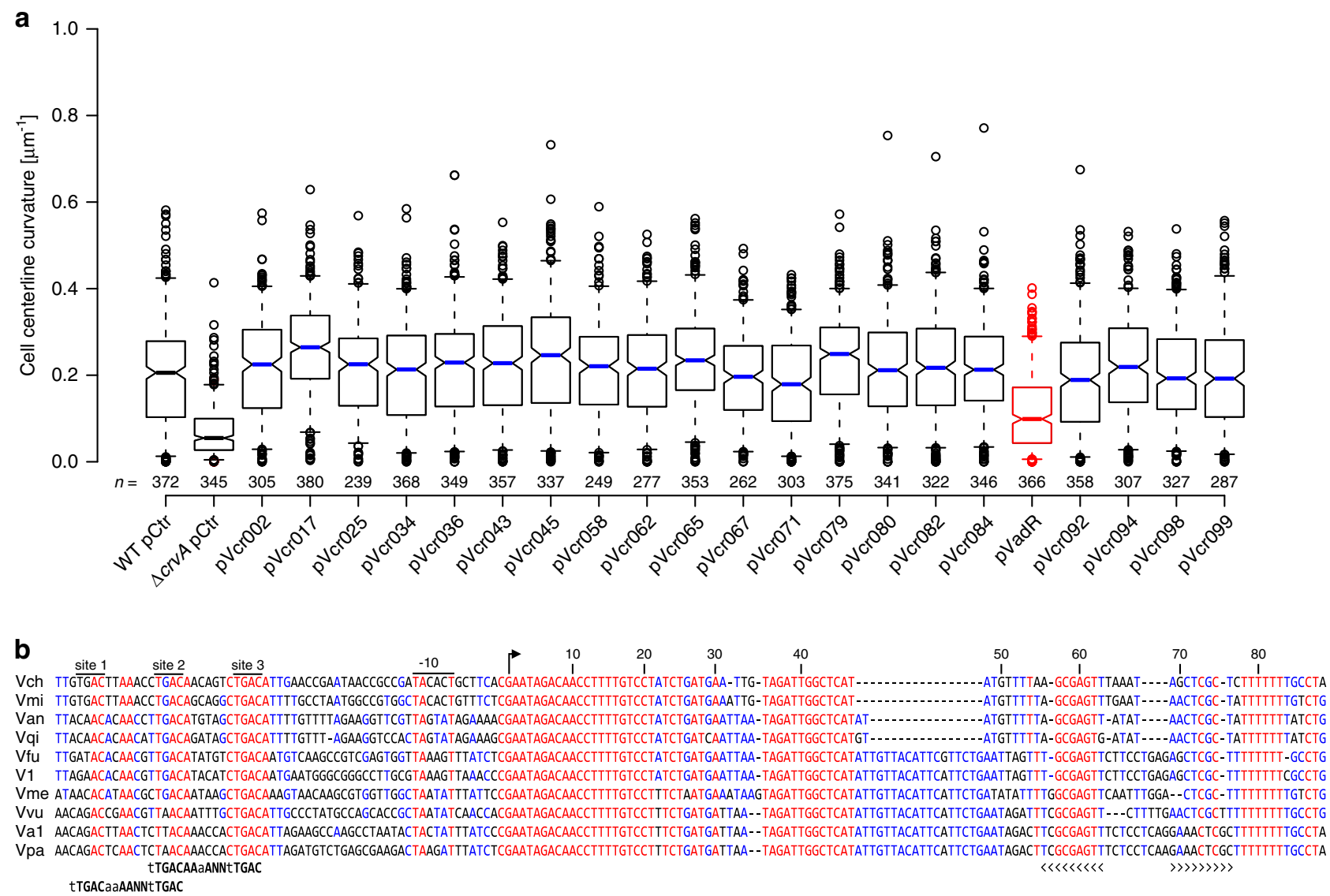

C

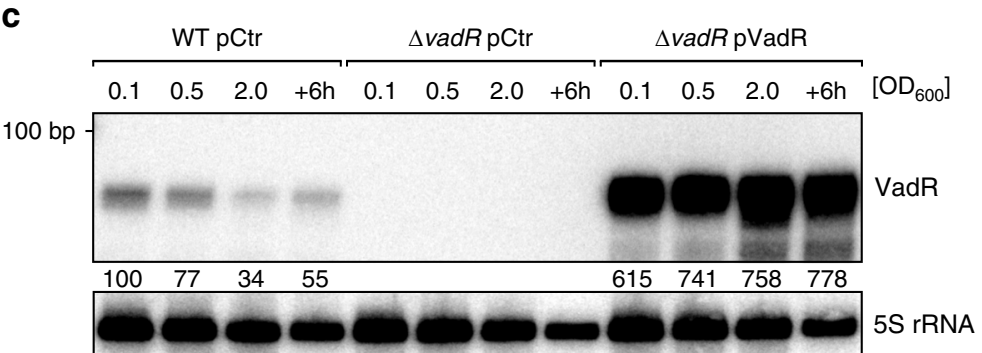

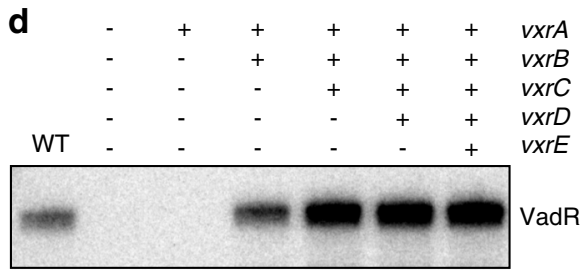

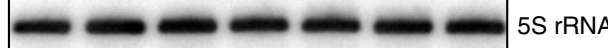

Fig. 1 The VadR sRNA alters $\boldsymbol{V}$. cholerae cell shape and is activated by VxrB. a Centerline curvature of $V$. cholerae cells expressing the indicated sRNAs ( $x$-axis). The blue, black and red lines indicate the median, boxes represent 25th and 75th percentiles, whiskers represent 5th and 95th percentiles and notches indicate $95 \%$ confidence intervals for each median. $n$ of each set is listed above the $x$-axis over three independent experiments. $\mathbf{b}$ Alignment of vadR and its promoter sequence from various Vibrio species (see "Methods" section "Sequence alignment" for details). The -10 box, TSS (arrow) and the Rho-independent terminator (brackets) are indicated. Putative $\mathrm{VxrB}$ binding sites and binding motifs (bold) are illustrated. $\mathbf{c}$ VadR expression throughout bacterial growth was monitored on Northern blots. V. cholerae wild-type or vadR mutant cells carrying either a control plasmid ( $\mathrm{pCtr}$ ) or a constitutive vadR overexpression plasmid ( $\mathrm{V} V \mathrm{adR}$ ) were tested. A quantification of $\mathrm{VadR}$ expression relative to the wild-type (at $O D_{600}$ of 0.1 ) is provided. $\mathbf{d} V$. cholerae $\triangle \operatorname{vxr} A B C D E$ cells were complemented with various cistrons of the vxrABCDE operon and tested for VadR expression on Northern blots. Expression of the $v_{x} A B C D E$ fragments was driven by the inducible pBAD promoter $(0.02 \%$ L-arabinose final conc.) and exponentially growing cells were harvested (OD 600 of 0.2 ). A $V$. cholerae wild-type strain harboring an empty vector served as control. The experiment was performed with three independent biological replicates $(n=3)$. Source data underlying panels $\mathbf{a}, \mathbf{c}$, and $\mathbf{d}$ are provided as a Source Data file.

first gene of all regulated operons; Supplementary Fig. 2a). The majority of repressed targets (15) corresponded to a single biofilm gene cluster ( $v c 0916-v c 0939)$ required for the production of the VPS biofilm exopolysaccharide, as well as genes producing the auxiliary biofilm components, RbmA-F ${ }^{23}$ (Fig. 2b).

Gene ontology (GO) analyses revealed a significant overrepresentation of GO terms associated with polysaccharide synthesis in the downregulated targets (Fig. 2c). Indeed, using the wrinkly colony morphology phenotype of $V$. cholerae hapR deficient cells as a read-out for biofilm formation ${ }^{20}$, we discovered that VadR overexpression resulted in strongly decreased biofilm formation (Fig. 2d). This phenotype was further corroborated by quantitative measurements of biofilm formation of $V$. cholerae $\triangle h a p R$ cells using microfluidic flow chambers and confocal microscopy. Analysis of the respective microscopic images revealed that $\mathrm{VadR}$ overexpression resulted in reduced biofilm density (Fig. 2e), and we were able to confirm this phenotype in biofilms formed by $V$. cholerae wild-type cells (Fig. 2f). Reduced biofilm density was previously reported for $V$. cholerae cells lacking the RbmA biofilm matrix protein ${ }^{19,24,25}$ and in accordance with these results and our transcriptomic experiments (Fig. 2a and Supplementary Fig. 2a), we discovered that VadR 
a

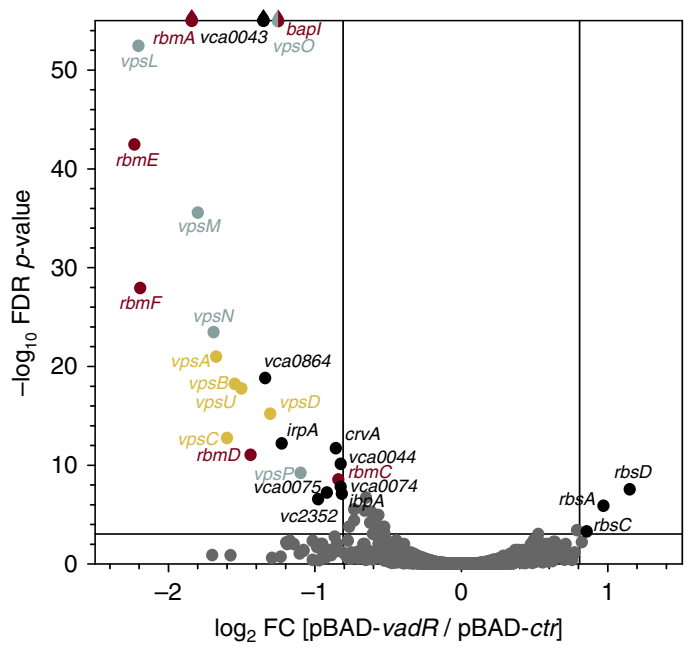

b

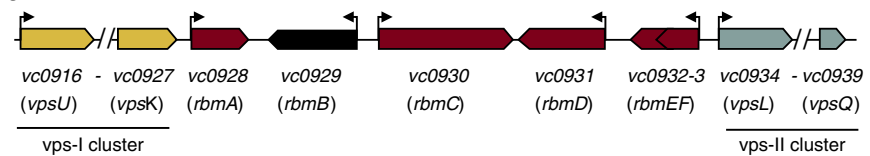

C

Polysaccharide biosynthetic process

Polysaccharide metabolic process

Carbohydrate biosynthetic process
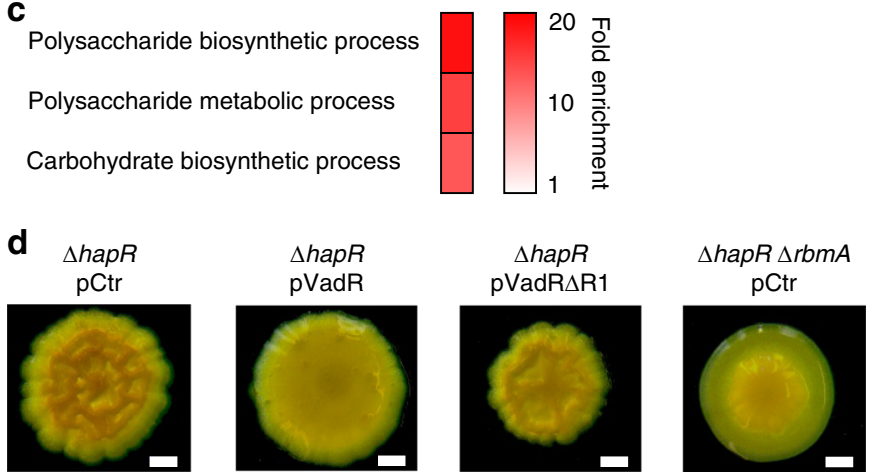

f

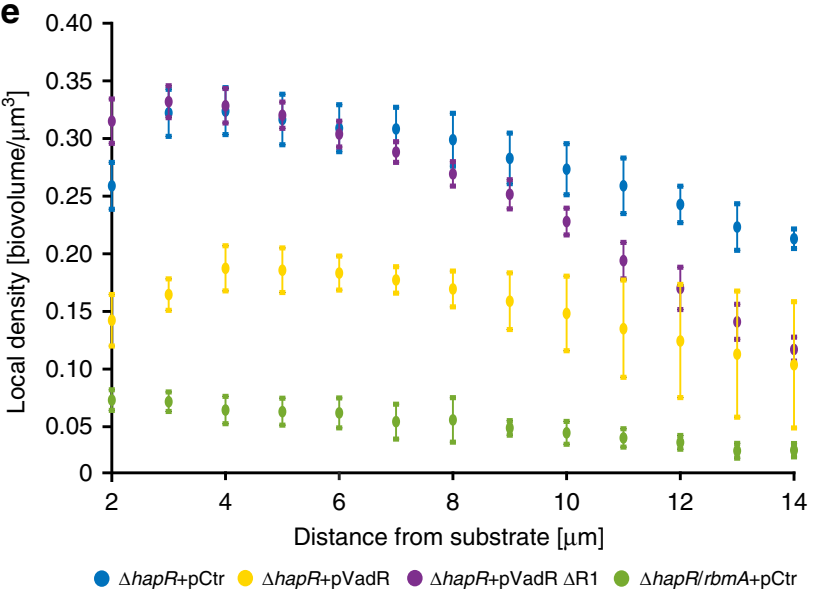

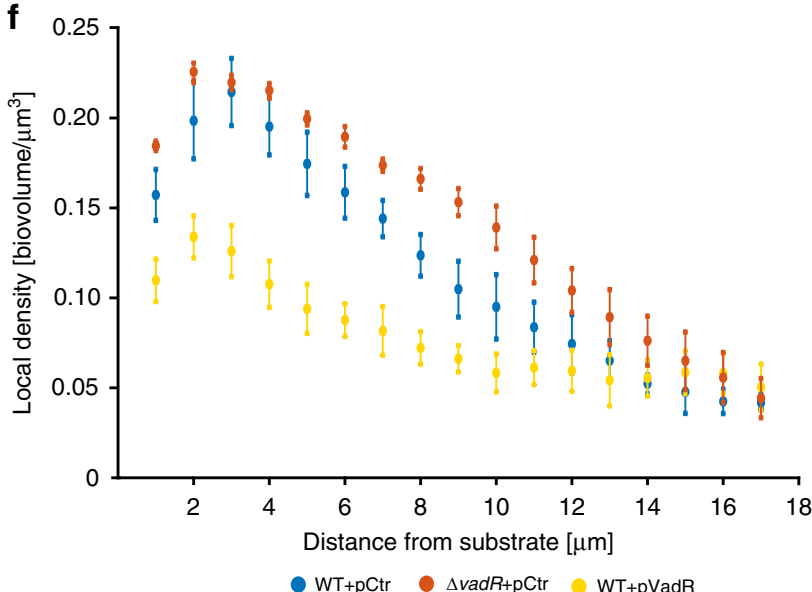

Fig. 2 Target spectrum of VadR and its role in biofilm formation. a Volcano plot analysis showing differentially regulated genes after pulse induction of VadR. Genes with absolute fold changes $\geq 1.75$ and an FDR (false discovery rate) corrected $p$-value of $\leq 0.001$ were considered significantly expressed and are indicated (FC, fold change). $\mathbf{b}$ Genomic context of the major biofilm cluster in $V$. cholerae. c Gene enrichment analysis of the differentially expressed genes shown in (a) using gene ontology analysis ${ }^{59}$. d Colony biofilm images of $V$. cholerae $\Delta$ hap $R$ and $\Delta h a p R / \Delta r b m A$ cells carrying the indicated plasmids. Each strain was spotted on LB agar plates and incubated for $48 \mathrm{~h}$ at room temperature before imaging. Representative images of two independent experiments are shown. Scale bars $=0.5 \mathrm{~mm}$. e Confocal spinning disk microscopy was used to monitor biofilm density of $V$. cholerae $\Delta$ hap $R$ cells carrying the indicated plasmids, as well as $\Delta h a p R / \Delta r b m A$ mutant harboring a control plasmid. Local cell density as a function of distance from the substratum was plotted for each of the indicated strains from three biologically independent experiments as mean \pm SD using the BiofilmQ software ${ }^{55}$. $\mathbf{f}$ Experiments analogous to $\mathbf{e}$, however, $V$. cholerae wild-type and $\Delta$ vadR cells (carrying the indicated plasmids) were monitored for biofilm density from three biologically independent experiments as mean \pm SD. Source data underlying panels $\mathbf{e}$ and $\mathbf{f}$ are provided as a Source Data file.

overexpression led to a $\sim 5$-fold decrease in RbmA levels, while $\operatorname{vadR}$ deletion mildly increased the production of the protein (Supplementary Fig. 2b). Similarly, we observed that $V$. cholerae $\Delta v a d R$ mutants displayed slightly increased biofilm densities when compared to wild-type cells. These results show that in addition to controlling cell shape, VadR also modulates biofilm formation in $V$. cholerae.

VadR acts post-transcriptionally to repress target gene expression. To investigate the molecular underpinnings of VadRmediated gene control in $V$. cholerae, we cloned the $5^{\prime}$ UTR (untranslated region) and the TIR (translation initiation region) of the 14 potential VadR targets into a GFP-based reporter plasmid designed to score post-transcriptional control ${ }^{26}$. Cotransformation of these plasmids with a VadR overexpression vector or a control plasmid in $E$. coli confirmed posttranscriptional repression of nine targets $(\operatorname{crvA}, \operatorname{irp} A, \operatorname{rbm} A$, $r b m D, v p s L, v p s U, v c 2352, v c a 0075$, and $v c a 0864$ ), while we were unable to validate direct regulation of bapI, $r b m C, r b m F, r b s D$, and vca0043 (Fig. 3a and Supplementary Fig. 3a). We currently do not know why we were unable to confirm regulation of these genes in E. coli, however, it is possible that VadR forms RNA duplexes with the deep coding sequence of their respective mRNAs, which would not be captured in our GFP reporters. Using the RNA hybrid algorithm ${ }^{27}$, we next predicted RNA duplex formations of VadR with $c r v A, \operatorname{rbmA}, v p s U$, and $v p s L$ (Fig. $3 b-e$ ). In all four cases, the potential pairing involved the target's TIR and sequence elements located in the first 30 nucleotides of VadR. Using compensatory base-pair exchange experiments (creating mutants M1, M2, and M3 in $v a d R$, see Supplementary Figs. $1 \mathrm{a}$ and $3 \mathrm{~b}$ ), we validated binding at the 


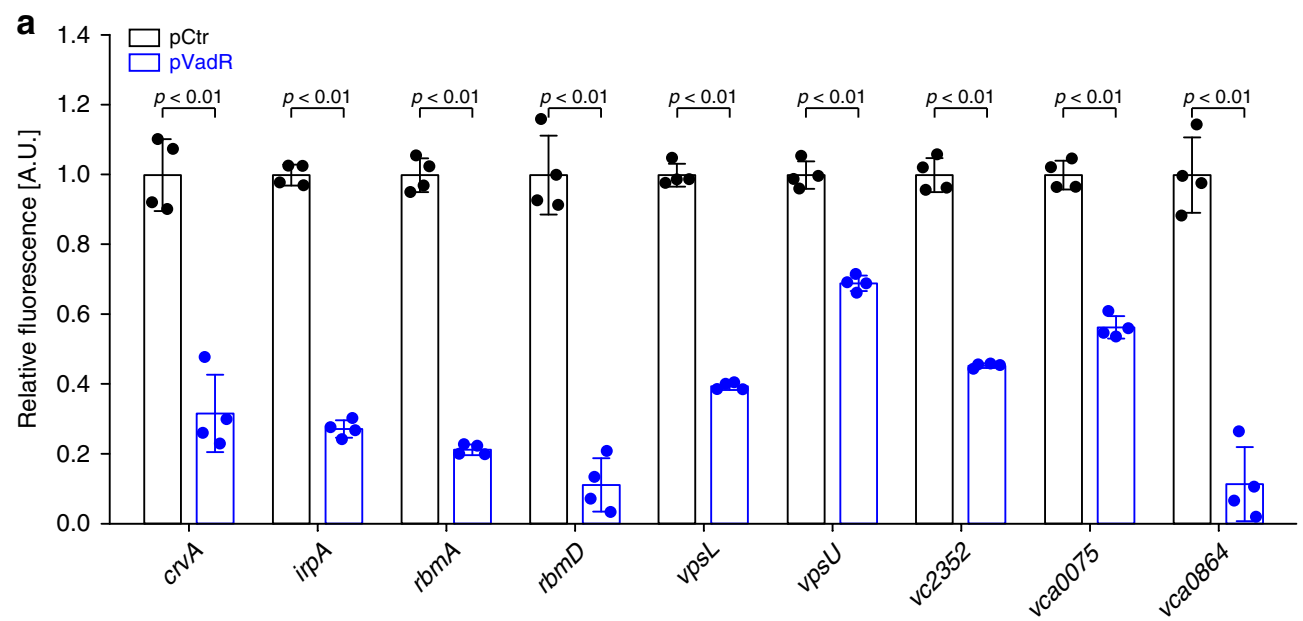

b
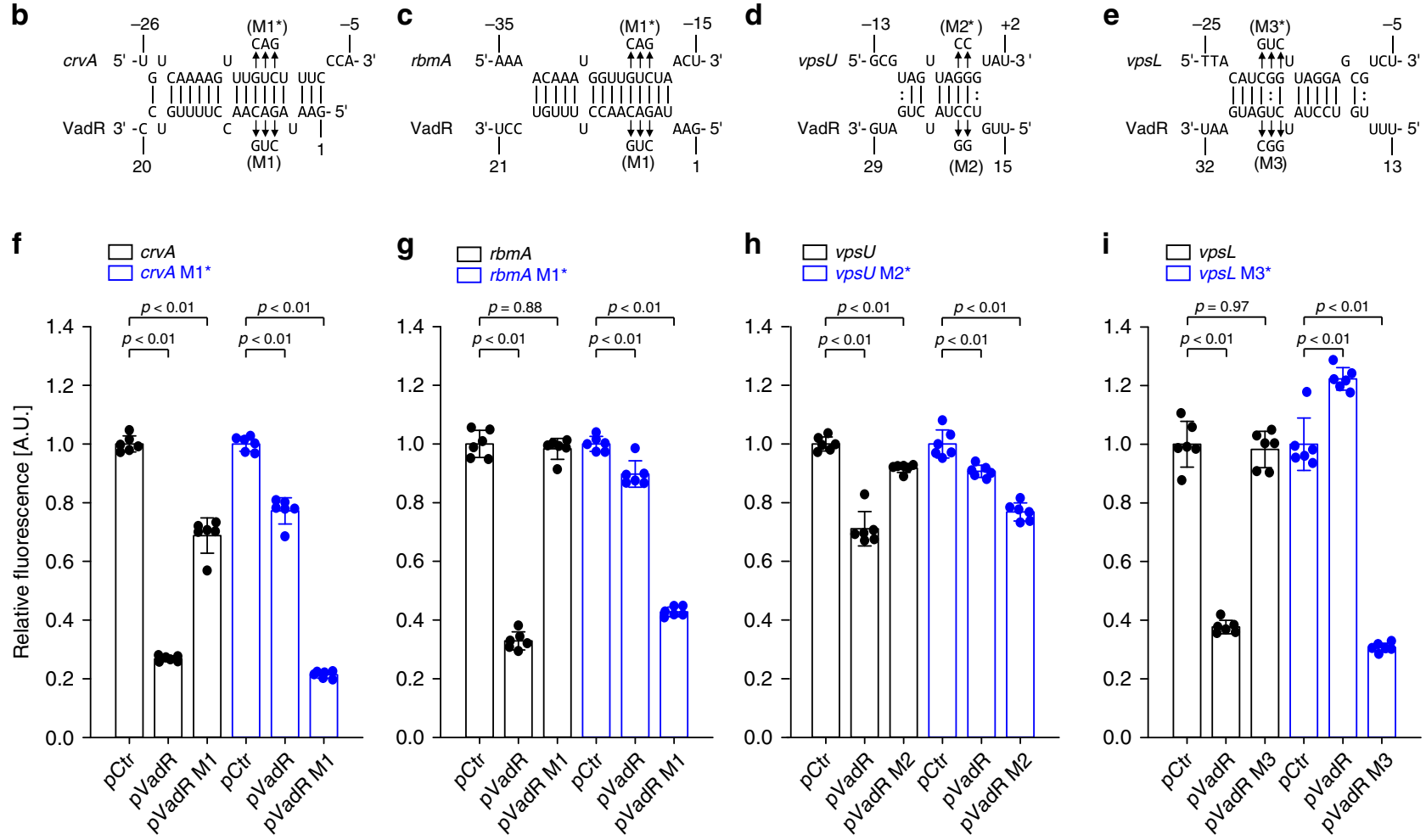

Fig. 3 VadR is a direct inhibitor of crvA and key biofilm transcripts. a Genes post-transcriptionally regulated by VadR. Fluorescence intensities of $E$. coli strains carrying the gene-specific reporters and the control plasmid ( $p C t r$ ) were set to 1 . Bars show mean of biologically independent replicates $\pm S D$, $n=4$. Statistical significance was determined using one-way ANOVA and post-hoc Sidak tests b-e Prediction of RNA duplex formation between selected mRNAs and VadR. Numbers indicate the distances from the TSS for VadR and the start codons of the target mRNA sequences, respectively. Arrows indicate the mutations tested in $\mathbf{f}-\mathbf{i}$. f-i Validation of the predicted mRNA-sRNA duplexes shown in $\mathbf{b}$-e using compensatory base-pair mutations. Fluorescence levels of E. coli strains harboring an empty vector control ( $\mathrm{pCtr}$ ) were set to 1 . Bars show mean of biologically independent replicates $\pm S D, n=6$. Statistical significance was determined using one-way ANOVA and post-hoc Sidak tests. Source data underlying panels $\mathbf{a}$ and $\mathbf{f}-\mathbf{i}$ are provided as a Source Data file.

predicted positions (Fig. $3 \mathrm{f}-\mathrm{i}$ ). To bolster these results at the phenotypic level, we also tested the effect of a VadR variant (VadR $\Delta \mathrm{R} 1$, see Supplementary Figs. 1a and $3 \mathrm{~b}$ ) unable to repress three of the four target genes on biofilm formation of hapRdeficient $V$. cholerae. In contrast to wild-type $\operatorname{VadR}, \operatorname{VadR} \Delta \mathrm{R} 1$ did not affect biofilm architecture (Fig. 2d, e), suggesting that VadR acts post-transcriptionally to modulate biofilm formation in V. cholerae.

VadR controls cell shape by inhibiting CrvA production. Our previous data revealed VadR as a direct repressor of the crvA mRNA (Fig. 3b, f) and hence we next aimed to study the role of
VadR in cell curvature in $V$. cholerae. Western blot analysis showed that CrvA levels were $\sim 1.5$-fold elevated in $\Delta v a d R$ cells, whereas VadR overexpression led to a $\sim 2$-fold reduction in CrvA production (Fig. 4a). We correlated these results with microscopic curvature analyses of single cells and discovered that vadR-deficient mutants displayed increased curvature, whereas plasmidborne VadR production had the reverse effect (Fig. 4b, c top). This effect was further amplified when cells were treated with sub-inhibitory concentrations of cefalexin forcing filamentation in $V$. cholerae (Fig. 4b, bottom). Importantly, neither vadR deletion, nor its overexpression affected cell length or volume of $V$. cholerae (Supplementary Fig. 4a, b), indicating that 

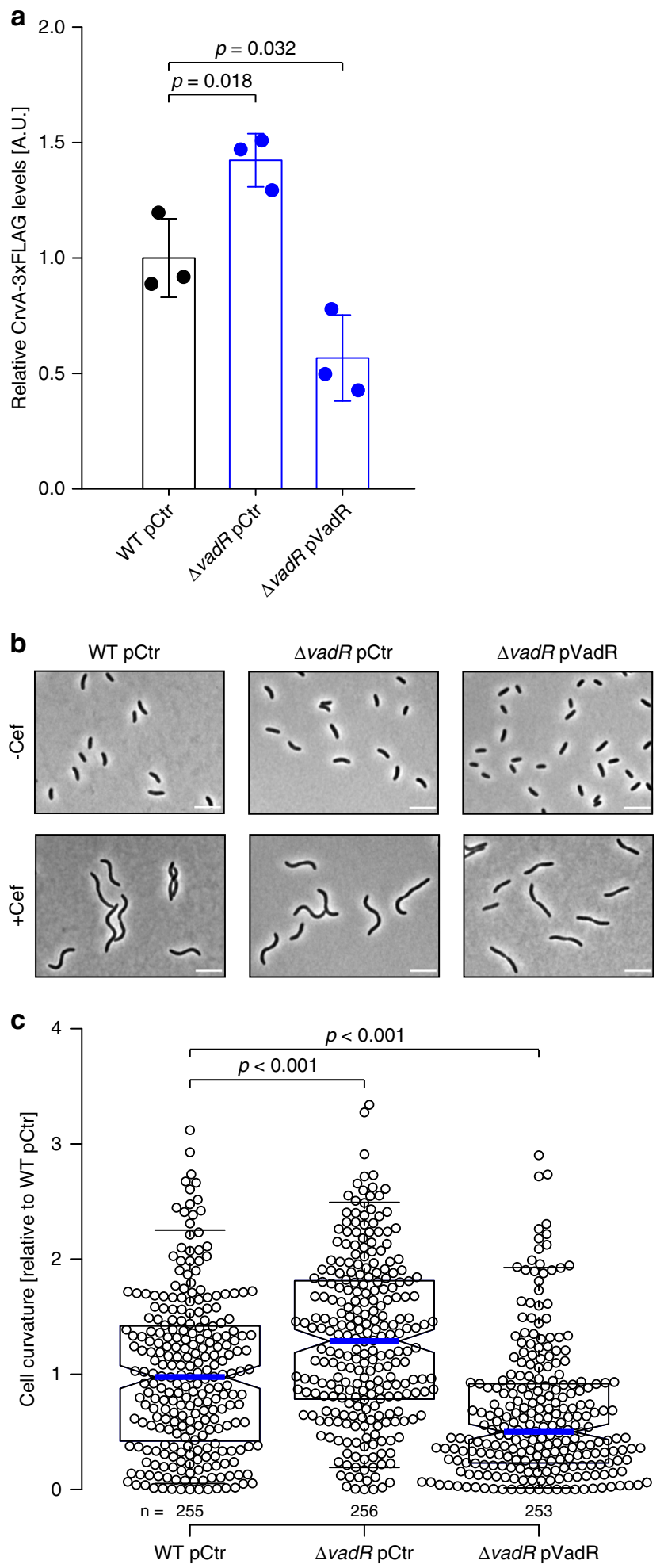

VadR specifically modulates cell curvature by inhibiting crvA expression.

VadR-mediated repression of $\boldsymbol{c r v A}$ affects antibiotic resistance. CrvA regulates cell curvature by spatially modulating peptidoglycan insertion in $V$. cholerae ${ }^{6}$ and the $\mathrm{VxrAB}$ regulon is induced by peptidoglycan-targeting antibiotics such as penicillin $\mathrm{G}^{18}$. Consequently, we tested the effect of penicillin $\mathrm{G}$ on VadR expression. Indeed, Northern blot analysis showed $\sim 7$-fold increased VadR levels in $V$. cholerae wild-type cells following
Fig. 4 VadR modulates V. cholerae curvature by repressing CrvA.

a Quantification of CrvA-3xFLAG protein levels in V. cholerae wild-type and vadR-deficient cells (carrying the indicated plasmids). Total protein samples of the indicated strains were harvested $\left(\mathrm{OD}_{600}\right.$ of 0.5$)$ and tested by Western blot analysis. CrvA-3xFLAG protein levels detected in the wildtype cells were set to 1 . Bars show mean of biological replicates $\pm S D, n=3$. Statistical significance was determined using one-way ANOVA and posthoc Holm-Sidak test. Samples of the three biologically independent replicates were processed in parallel. b Microscopy of cells used in (a-Cef). A second set of cells was treated with cefalexin for $1 \mathrm{~h}(+\mathrm{Cef})$ after reaching an $\mathrm{OD}_{600}$ of 0.5 . Shown are representative fields of vision (biological replicates $n=3$ ). Scale bars $=5 \mu \mathrm{m}$. c Analysis of cell centerline curvature in -Cef samples of $\mathbf{b}$. The curvature mean of wild-type cells was set to 1 . A blue line indicates the median, boxes represent 25 th and 75 th percentiles, whiskers represent 5th and 95th percentiles and notches indicate $95 \%$ confidence intervals for each median. $n$ of each set is listed above the $x$-axis over three independent experiments. Statistical significance was determined using one-sided Kruskal-Wallis test and posthoc Dunn's test. Source data underlying panels a and $\mathbf{c}$ are provided as a Source Data file.

treatment with penicillin G (Fig. 5 a) and we observed $\sim 25$-fold induction when we tested $v a d R$ promoter activity using a transcriptional reporter (Fig. 5b). In both cases, penicillin Gdependent activation of $v a d R$ was abrogated in the $\triangle v x r A B C D E$ strain (Fig. 5a, b). Expression of $\operatorname{vadR}$ was also activated by the MreB-targeting antibiotic A22 28 , albeit to a lower extent when compared to penicillin G (Supplementary Fig. 5a).

Based on these results, we speculated that modulation of cell shape by $\operatorname{VrxAB}$ and $\operatorname{VadR}$ might affect the sensitivity of $V$. cholerae towards cell-wall damaging antibiotics. Following this hypothesis, we first determined the effect of penicillin G treatment on VadR-mediated CrvA repression. Indeed, following treatment of $V$. cholerae with penicillin G, we discovered $\sim 3.5-$ fold higher CrvA levels in $\Delta v a d R$ cells when compared to wildtype $V$. cholerae (Supplementary Fig. 5b). In addition, we also discovered reduced penicillin $G$ survival rates for $v a d R$-deficient $V$. cholerae cells and we were able to complement this phenotype using plasmid-borne VadR production (Fig. 5c). To pinpoint this effect to VadR-mediated repression of crvA in the presence of penicillin G, we introduced mutation $\mathrm{M}^{*}$ (Fig. $3 \mathrm{~b}$ ) at the chromosomal crvA locus. This mutation does not abrogate $c r v A$ expression (Supplementary Fig. 5c), but renders the transcript immune towards post-transcriptional repression by VadR. This strain phenocopied the effect of a $v a d R$ mutant and we obtained almost identical results when we introduced the corresponding mutation (M1, Fig. 3b and Supplementary Fig. 1a) at the chromosomal vadR gene (Fig. 5c). Combination of the two mutant alleles resulted in a partial restoration of penicillin $G$ resistance (Fig. 5c), indicating that VadR might be required to mitigate the detrimental effect of CrvA under antibiotic pressure. Notably, neither mutation nor overexpression of $v a d R$ affected survival of $V$. cholerae under standard growth conditions (Supplementary Fig. 5d).

VadR is expressed during early biofilm formation. To connect the roles of VadR in cell curvature regulation and biofilm formation, we monitored VadR expression (using a PvadR::mRuby2 transcriptional reporter) in growing biofilms of $V$. cholerae wildtype cells employing single-cell confocal microscopy analysis ${ }^{29}$. When normalized for sfGFP production driven from the constitutive $P_{\text {tac }}$ promoter, we discovered that the $v a d R$ promoter is most active during the initial phases of biofilm formation, while expression is switched off in mature biofilms (Fig. 6a). In parallel, 
a
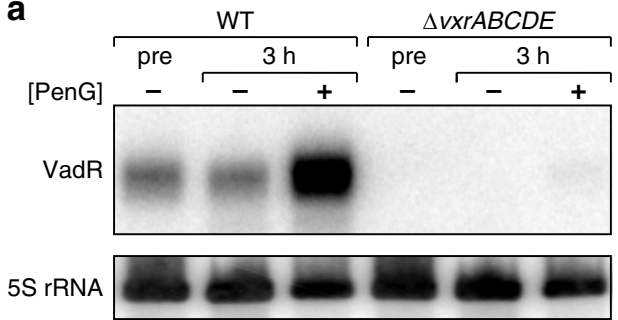

b
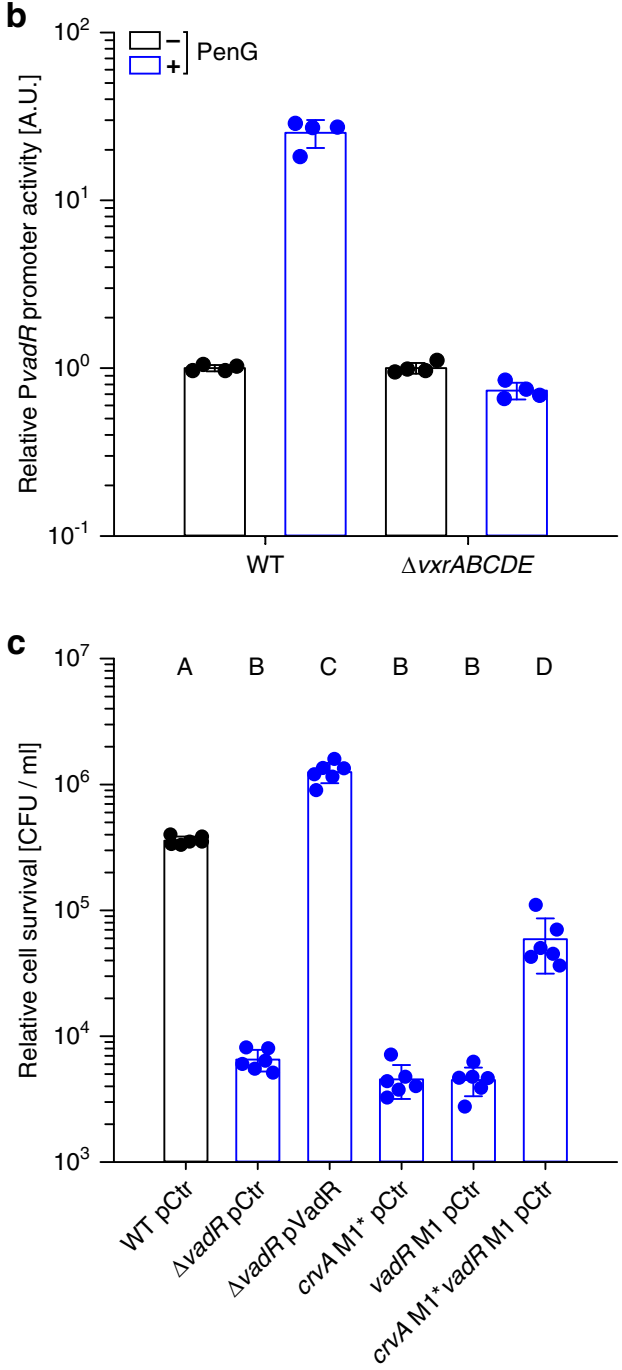

Fig. 5 VadR mediates B-lactam resistance through repression of crvA. a $V$. cholerae wild-type and $v x r A B C D E$ mutant strains were grown to $O D=$ 0.2 (pre) and split into two sets. One set was treated with penicillin $\mathrm{G}$, while the other set received a mock treatment. After $3 \mathrm{~h}$, RNA was isolated and $\mathrm{VadR}$ expression was monitored by Northern analysis. b $\mathrm{VadR}$ promoter activity was tested under the same conditions as in $\mathbf{a}$ using a fluorescent transcriptional reporter. Promoter activities of mock-treated strains were set to 1 . Bars represent mean of biologically independent replicates $\pm S D, n=4$. c The indicated $V$. cholerae strains ( $x$-axis) were grown to $\mathrm{OD}_{600}=0.4$ and treated with penicillin $\mathrm{G}$ for $3 \mathrm{~h}$. Survival after treatment was determined by counting colony forming units (CFUs). Bars represent mean of biologically independent replicates $\pm S D, n=6$.

Statistical significance was determined using one-way ANOVA and posthoc Holm-Sidak test. Significantly different groups $(p<0.01)$ are labeled with corresponding letters. Source data underlying panels $\mathbf{a}-\mathbf{c}$ are provided as a Source Data file. we also determined cell curvature of individual cells during biofilm development (Fig. 6b). Comparison of the two datasets showed that VadR expression and cell curvature are negatively correlated (Fig. 6c), suggesting that VadR expression results in straighter cells during early phases of biofilm development, whereas mature biofilms are more likely to contain a higher proportion of curved cells.

\section{Discussion}

There is an ever growing list of microbial functions and phenotypes involving RNA-based gene regulation. Here, we have identified the Hfq-dependent VadR sRNA as regulator of cell shape and biofilm formation in $V$. cholerae. While sRNAmediated control of microbial cell shape was previously unknown, other sRNAs have been reported to modulate biofilm formation in $V$. cholerae and other species ${ }^{30}$. For example, we have previously shown that the VqmR sRNA also inhibits biofilm formation in $V$. cholerae ${ }^{20,31}$, however, instead of base-pairing with the mRNAs of the multiple biofilm components (as shown here for VadR), VqmR inhibits translation of $v p s T$, encoding a transcriptional activator of biofilm genes in $V$. cholerae ${ }^{32}$. Thus, although VadR and VqmR both inhibit biofilm formation, VqmR acts at a higher level of the biofilm pathway. This difference in regulatory hierarchy could also point to a conceptual difference in the underlying regulatory principle of how these two sRNAs fulfill their function in the cell. Repression of $v p s T$ translation by VqmR will become effective when VpsT protein levels decreased sufficiently to affect the transcription of biofilm-related genes and thus might be considered to be part of larger regulatory process that mediates the switch from low-cell to high-cell density behaviors in $V$. cholerae ${ }^{9}$. In contrast, inhibition of multiple biofilm genes by VadR should produce a more immediate response to adjust the production of biofilm factors to the status of $\operatorname{VxrAB}$ system.

Interestingly, the $\mathrm{VxrAB}$ system has previously been shown to induce biofilm formation in $V$. cholerae $^{33}$, which at first glance might seem counterintuitive given that VadR is also induced by $\mathrm{VxrAB}$ and inhibits biofilm formation. Nevertheless, bacterial sRNAs are known to be frequently part of mixed regulatory networks involving transcriptional regulators as well as noncoding RNAs ${ }^{34}$. It is currently unclear at which level $\mathrm{VxrAB}$ function to activate biofilm formation, however, overexpression of $\mathrm{VxrB}$ induces the $v p s L$ promoter ${ }^{33}$ and the $v p s L$ mRNA is repressed by VadR (Fig. 3i). It is therefore possible that VxrB, VadR, and $v p s L$ form a type 1 incoherent feed-forward loop (I1-FFL), in which the top activator regulates both a gene and a repressor of the gene. I1-FFL have been reported to create pulse-like gene expression dynamics and to accelerate the response time of a system ${ }^{35}$. In addition, I1-FFL can reduce gene expression noise ${ }^{36}$, which is particularly relevant when the activity of the upstream transcriptional activator (here $\mathrm{VxrB}$ ) fluctuates. The exact signal triggering the $\operatorname{Vxr} A B$ system is currently unknown, however, in the closely related species Vibrio parahaemolyticus, the VxrAB system (a.k.a. VbrKR) has been reported to respond to B-lactam antibiotics via direct interaction with the histidine kinase, $\mathrm{VbrK}^{37}$. Our results support activation of the system by ß-lactam antibiotics, i.e., penicillin $\mathrm{G}$ (Fig. 5a, b), however, since we also discovered $\mathrm{vadR}$ activation in the presence of A22 (Supplementary Fig. 5a), it is likely that additional cues also trigger the system.

In fact, VadR expression is readily detectable under standard growth conditions (Fig. 1c) suggesting a regulatory role for the system under non-stress conditions. Here, VadR might take the role of adjusting cell growth with the production of CrvA and biofilm-forming factors (Fig. 6d). CrvA is an abundant periplasmic protein ${ }^{6}$ and biofilm components require transport 
a

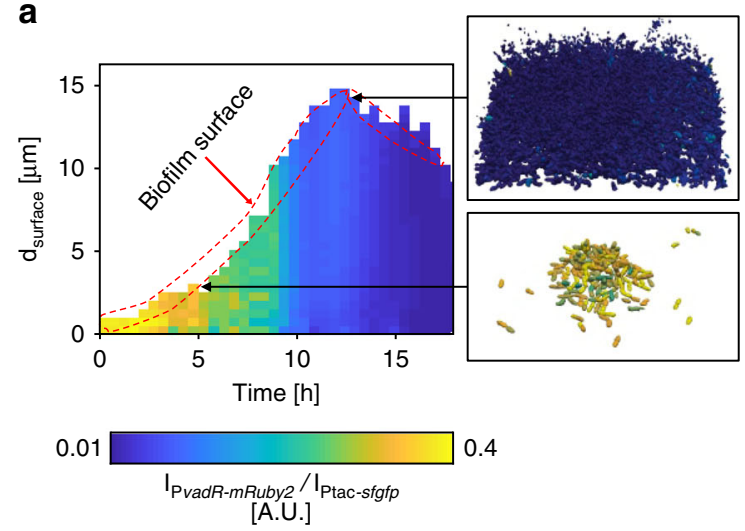

b

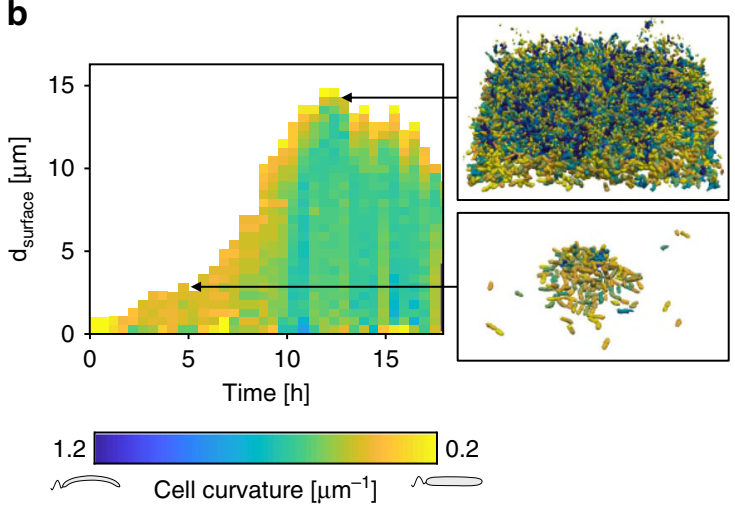

d
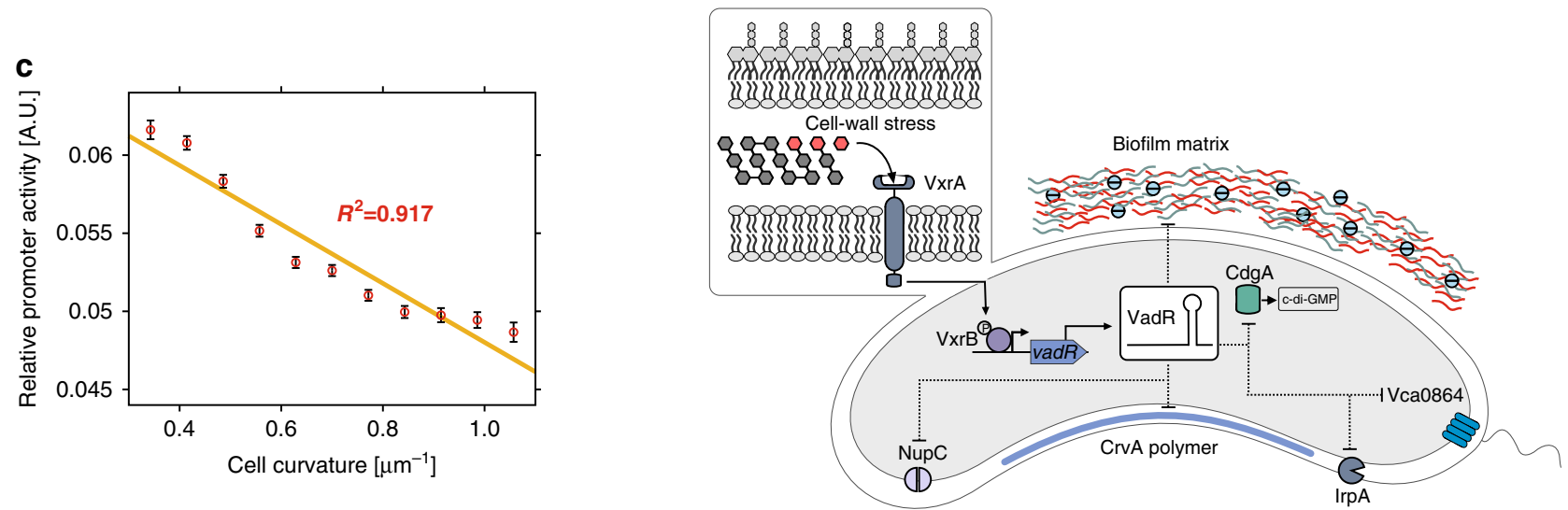

Fig. 6 VadR controls cell curvature during biofilm development. a Relative activity of the vadR promoter during biofilm growth of wild-type $V$. cholerae cells. In each cell the fluorescence of mRuby2, expressed from the vadR promoter was normalized by the signal of the constitutive Ptac-promoter-driven sfGFP-fluorescence signal. Heatmap shows vadR promoter activity at both spatial (distance from surface of biofilm representing height of the biofilm) and temporal (time of biofilm growth) resolution. Subset of images show the cells from two time points and separate locations of the biofilm. These cells were rendered by ParaView ${ }^{56}$ after final segmentation and analysis using BiofilmQ ${ }^{55}$. The color of each cell represents the activity of the vadR promoter. b Spatio-temporal heatmap showing cell curvature of each cell for $V$. cholerae biofilms. Cell curvature of individual cells was calculated using BiofilmQ ${ }^{55}$. To calculate the cell curvature of each cell inside the biofilms, similar positions of the biofilm as in a were selected for rendering. In these subset of images, the color represents the cell curvature of each cell. c A correlation graph was plotted for vadR promoter activity as function of cell curvature. Calculation of vadR promoter activity and cell curvature was done for $V$. cholerae wild-type biofilms grown in flow chambers. Each point represents the mean \pm SEM of $>1000$ cells for given time point in a biofilm. d Model showing the regulatory functions of the VadR sRNA in $V$. cholerae. Expression of vadR sRNA is controlled by the $\operatorname{Vx} A B$ two-component system. The sRNA regulates multiple biological processes, including cell shape and biofilm formation. Source data underlying panels a-c are provided as a Source Data file.

across two membranes to reach their final destination ${ }^{23}$. Uncoordinated export of proteins and polysaccharides can clog the cellular transport machineries and compromise the permeability barrier or structural integrity of the cell ${ }^{38,39}$. It is therefore vital for the cell to synchronize these functions with cell growth and sRNAs have previously been implicated in this process ${ }^{40}$. For example, sRNAs activated by the alternative sigma-factor E promote envelope homeostasis by tuning the levels of newly synthesized outer membrane proteins in response to misfolded proteins in the periplasm $8,21,41,42$. VadR could take an analogous position in the $\operatorname{VxrAB}$ stress response system and given the relatively short half-life of VadR ( 3 min, Supplementary Fig. 1c), sRNA-based regulation might provide regulatory dynamics that are superior over canonical protein-based regulation ${ }^{14}$.

How CrvA affects peptidoglycan remodeling in $V$. cholerae is currently not fully understood. Previous reports indicated that filament-like proteins such as CrvA and CreS render the activity of enzymes involved in cell-wall synthesis and thereby reduce the rate of peptidoglycan insertion at one site of the cell ${ }^{6,43}$. This process results in asymmetric growth and cell curvature. Indeed, when comparing incorporation of HADA (HCC-amino-D-alanine ${ }^{44}$ ) into peptidoglycan of $V$. cholerae wild-type and VadR-overexpressing cells, we observed that HADA insertion was biased towards the outer surface of wild-type cells, while VadR-overexpression restored this effect (Supplementary Fig. 6a). Given that CrvA does not affect the overall peptidoglycan composition in $V$. cholerae $^{6}$, it might be possible that the relative increase in peptidoglycan synthesis at the outer surface facilitates the activity of $B$-lactam antibiotics at this side and thus stimulates cell wall lysis. This idea is supported by our finding that penicillin-treated $V$. cholerae wild-type cells are more prone to form spheroplasts, when compared to VadRoverexpressing cells (Supplementary Fig. 6b). Taken together, VadR-mediated repression of crvA mRNA could help to mitigate curvature-associated antibiotic sensitivity by reducing the de novo production of CrvA protein under stress conditions.

While adjusting cell curvature in response to cell growth and antibiotic stress could be an important feature of VadR-mediated gene regulation in $V$. cholerae, it is relevant to note that not all Vibrios encode the $c r v A$ gene (e.g., $V$. parahaemolyticus) and thus do not display a curved cell morphology 6 . However, vadR and $\operatorname{vxr} A B$ are highly conserved in these organisms (Fig. $1 \mathrm{~b}$ and Supplementary Fig. 7) suggesting that VadR's role goes beyond 
cell curvature control. The $v p s L$ and $v p s U$ genes are highly conserved among the biofilm-associated factors repressed by VadR, while $r b m A$ and $r b m D$ show only poor conservation (Supplementary Fig. 7). Additional targets of VadR are $v c 2352$ (encoding a NupC-type nucleoside transporter ${ }^{45}$ ), $\operatorname{irp} A$ (encoding an ironregulated membrane protein carrying a peptidase domain ${ }^{46}$ ), vca0864 (encoding a methyl-accepting chemotaxis protein ${ }^{47}$ ), and $v c a 0075$ (function unknown ${ }^{48}$ ), all of which display high conservation among the Vibrios (Figs. 3a, 6d and Supplementary Fig. 7). Interestingly, $v c a 0075$ is co-repressed with $c d g A^{20}$, a diguanylate cyclase gene with documented functions in biofilm formation ${ }^{49}$. In addition, Vca0864 has been reported to inhibit chemotaxis towards $\mathrm{N}$-acetylglucosamine, which is a key for peptidoglycan synthesis ${ }^{50}$. We currently do not yet know how these genes fit into the VadR regulon, however, understanding their biological functions might help to obtain a more comprehensive view on the VadR regulon in $V$. cholerae and related Vibrios.

\section{Methods}

Bacterial strains and growth conditions. Bacterial strains used in this study are listed in Supplementary Table 2. Details for strain construction are provided in the Supplementary Material and Methods section. V. cholerae and E. coli cells were grown under aerobic conditions $\left(200 \mathrm{rpm}, 37^{\circ} \mathrm{C}\right)$ in $\mathrm{LB}$ (Lennox Broth). Where appropriate, media were supplemented with antibiotics at the following concentrations: $100 \mu \mathrm{g} \mathrm{mL}^{-1}$ ampicillin; $20 \mu \mathrm{g} \mathrm{mL}^{-1}$ chloramphenicol; $50 \mu \mathrm{g} \mathrm{mL}^{-1}$ kanamycin; $50 \mathrm{U} \mathrm{mL}^{-1}$ polymyxin $\mathrm{B} ; 5 \mathrm{mg} \mathrm{mL}^{-1}$ streptomycin, $5 \mu \mathrm{g} \mathrm{mL}^{-1}$ cefalexin, and $50 \mu \mathrm{g} \mathrm{mL}^{-1}$ penicillin $\mathrm{G}$.

Plasmids and DNA oligonucleotides. All plasmids and DNA oligonucleotides used in this study are listed in Supplementary Tables 3 and 4, respectively. Cloning details are provided in the Supplementary Material and Methods section.

RNA isolation and Northern blot analysis. Total RNA was prepared and blotted as described previously ${ }^{51}$. Membranes (GE Healthcare Amersham) were hybridized with $\left[{ }^{32} \mathrm{P}\right]$ labeled DNA oligonucleotides at $42{ }^{\circ} \mathrm{C}$. Signals were visualized using a Typhoon phosphorimager (GE Healthcare) and quantified using Gelquant software (biochemlabsolutions).

Screen for curvature-regulating sRNAs and microscopy analysis. V. cholerae wild-type cells were conjugated with either an empty control plasmid (pCMW-1K) or overexpression plasmids of the following sRNAs: Vcr002, Vcr017, Vcr025, Vcr034, Vcr036, Vcr043, Vcr045, Vcr058, Vcr062, Vcr065, Vcr067, Vcr071, Vcr079, Vcr080, Vcr082, Vcr084, VadR, Vcr092, Vcr094, Vcr098, or Vcr099. For microscopy analyses, the respective $V$. cholerae strains were cultivated in LB to $\mathrm{OD}_{600}$ of 0.4 . Cells were pelleted, washed in $1 \times \mathrm{PBS}$, and resuspended in $2.5 \%$ paraformaldehyde in $1 \times$ PBS. Phase contrast imaging was performed on a Zeiss Axio Imager M1 microscope equipped with EC Plan Neofluar $100 \times / 1.3$ Oil Ph3 objective (Zeiss). For further analyses, e.g., measurements of cell center line curvature, cell length and cell area, the FIJI-plugin MicrobeJ was used ${ }^{52,53}$. Colony biofilms were imaged with Stemi 305 stereoscopic microscope (Zeiss) and acquired with Labscope (Zeiss)

Flow chamber biofilms and confocal imaging. The strains were grown in LB medium supplemented with $50 \mu \mathrm{g} \mathrm{mL}^{-1}$ kanamycin, to mid-exponential growth phase, before introducing into microfluidic flow chambers. Flow chambers were constructed from poly(dimethylsiloxane) bonded to glass coverslips using an oxygen plasma. The microfluidic channels measured $500 \mu \mathrm{m}$ in width, $100 \mu \mathrm{m}$ in height and $7 \mathrm{~mm}$ in length. After the cultures were introduced into the channels, the channels were incubated at $24^{\circ} \mathrm{C}$ for $1 \mathrm{~h}$ without any flow, to allow cells to attach to the bottom glass surface of the channels. The flow was then set to 0.3 $\mu \mathrm{L} \mathrm{min}{ }^{-1}$ for approximately $18 \mathrm{~h}$ before images were acquired. Cells were stained with green fluorescent nucleic acid stain dye, SYTO 9 (Thermo Fisher Scientific), by exchanging the syringes containing LB with SYTO 9 for $30 \mathrm{~min}$. Flow rates were controlled using a high-precision syringe pump (Pico Plus, Harvard Apparatus). To acquire the spatiotemporal information of individual cells in a growing biofilm, time lapse confocal microscopy was performed as described previously ${ }^{54}$. To reduce photobleaching and phototoxicity during time-lapse imaging, a live feedback between image acquisition, image analysis and microscope control was used to automatically detect the biofilm height to avoid imaging of empty space on top of the biofilms. Images were acquired with an Olympus $100 \times$ objective with numerical aperture of 1.35 , using a Yokogawa spinning disk confocal scanner and laser excitation at $488 \mathrm{~nm}$. Images were acquired at spatial resolution of $63 \mathrm{~nm}$ in the $x y$-plane and $400 \mathrm{~nm}$ along the $z$ direction. To detect all single cells, measure cell curvature of each cell, and quantify the relative $v a d R$ promoter-reporter strength from biofilm grown in flow chambers, biofilm images were analysed using the BiofilmQ software ${ }^{55}$. Kymograph heatmaps showing the strength of $v a d R$ promoter and cell curvature during biofilm growth were generated with BiofilmQ. 3-D cell rendering was done using BiofilmQ-analysed biofilm data using the ParaView software ${ }^{56}$. Biofilm images were prepared with the NIS-Elements AR Analysis software (Nikon) by cropping a fixed $z$-plane with $x y$ and $y z$ projections.

RNA-seq analysis. Biological triplicates of $V$. cholerae $\Delta$ vadR strains harboring $\mathrm{pBAD}-\mathrm{Ctr}$ or $\mathrm{pBAD}-v a d R$ plasmids were grown to exponential phase $\left(\mathrm{OD}_{600}\right.$ of $0.2)$ in LB media. sRNA expression was induced by addition of L-arabinose $(0.2 \%$ final conc.). After $10 \mathrm{~min}$ of induction, cells were harvested by addition of 0.2 volumes of stop mix (95\% ethanol, 5\% (v/v) phenol) and snap-frozen in liquid nitrogen. Total RNA was isolated and digested with Turbo DNase (Thermo Fischer Scientific). Ribosomal RNA was depleted using Ribo-Zero kits (Epicenter) for Gram-negative bacteria, and RNA integrity was confirmed using a Bioanalyzer (Agilent). Directional cDNA libraries were prepared using the NEBNext Ultra II Directional RNA Library Prep Kit for Illumina (NEB, \#E7760). The libraries were sequenced using a HiSeq 1500 system in single-read mode for 100 cycles. The read files in FASTQ format were imported into CLC Genomics Workbench v11 (Qiagen) and trimmed for quality and $3^{\prime}$ adapters. Reads were mapped to the $V$. cholerae reference genome (NCBI accession numbers: NC_002505.1 and NC_002506.1) using the "RNA-Seq Analysis" tool with default parameters. Reads mapping to annotated coding sequences were counted, normalized (CPM) and transformed $\left(\log _{2}\right)$. Differential expression between the conditions was tested using the "Empirical Analysis of DGE" command. Genes with a fold change $\geq 1.75$ and an FDR adjusted $p$-value $\leq 0.001$ were defined as differentially expressed.

Fluorescence measurements. Fluorescence assays to measure GFP expression were performed as described previously ${ }^{26}$. E. coli strains expressing translational GFP-based reporter fusions were grown for $16 \mathrm{~h}$ in LB medium and resuspended in $1 \times$ PBS. Fluorescence intensity was quantified using a Spark $10 \mathrm{M}$ plate reader (Tecan). V. cholerae and E. coli strains carrying mKate 2 transcriptional reporters were grown in LB medium, resuspended in $1 \times$ PBS, samples were collected at the indicated time points and mKate2 fluorescence was measured using a Spark $10 \mathrm{M}$ plate reader (Tecan). Control samples not expressing fluorescent proteins were used to subtract background fluorescence.

Western blot analysis. Experiments were performed as previously described ${ }^{57}$ Protein samples were separated using SDS-PAGE and transferred to PVDF membranes for Western blot analysis. $3 \times$ FLAG-tagged fusions were detected using anti-FLAG antibody (Sigma, \#F1804). RnaPa served as a loading control and was detected using anti-RnaPa antibody (BioLegend, \#WP003). Signals were visualized using a Fusion FX EDGE imager (Vilber) and band intensities were quantified using the BIO-1D (Vilber) or Gelquant software (biochemlabsolutions).

Sequence alignment. VadR and its promoter sequences among various Vibrio species were aligned using the MultAlin webtool ${ }^{58}$. Vch: Vibrio cholerae (NCBI: txid243277), Vmi: Vibrio mimicus (NCBI:txid1267896), Van: Vibrio anguillarum (NCBI:txid55601), Vqi: Vibrio qinghaiensis (NCBI:txid2025808), Vfu: Vibrio furnissii (NCBI:txid29494), Vfl: Vibrio fluvialis (NCBI:txid676), Vme: Vibrio mediterranei (NCBI:txid689), Vvu: Vibrio vulnificus (NCBI:txid672), Val: Vibrio alginolyticus (NCBI:txid663), and Vpa: Vibrio parahaemolyticus (NCBI:txid670).

Statistical analyses. Statistical parameters for the respective experiment are indicated in the corresponding figure legends. $n$ represents the number of biological replicates. Details for the performed statistical tests are provided in the supporting information. Statistical analyses of CFUs were performed as follows: The data were $\log _{10}$-transformed and tested for normality and equal variance using Kolmogorov-Smirnov and Brown-Forsythe tests, respectively. The data were tested for significant differences using one-way ANOVA and posthoc Holm-Sidak tests. Significance levels are reported in the in the supporting information. Statistical analysis was performed using SigmaPlot v14 (Systat) and GraphPad Prism 8 (GraphPad Software). No blinding or randomization was used in the experiments. No estimation of statistical power was used before performing the experiments and no data were excluded from analysis.

Reporting summary. Further information on research design is available in the Nature Research Reporting Summary linked to this article.

\section{Data availability}

The raw data of the transcriptome analyses are available at the National Center for Biotechnology Information Gene Expression Omnibus (GEO) under the accession number GSE145764. Source data are provided with this paper. 


\section{Code availability}

The biofilm image analysis software tool BiofilmQ ${ }^{55}$ is available online (https:// drescherlab.org/data/biofilmQ/ and https://doi.org/10.1101/735423). HADA-profiles of outer and inner cell surfaces were determined by using a custom method, developed by FIJI. The respective code is part of yet unpublished work and available upon request.

Received: 18 March 2020; Accepted: 6 November 2020;

Published online: 27 November 2020

\section{References}

1. Taylor, J. A., Sichel, S. R. \& Salama, N. R. Bent bacteria: a comparison of cell shape mechanisms in proteobacteria. Annu. Rev. Microbiol. 73, 457-480 (2019).

2. Cabeen, M. T. \& Jacobs-Wagner, C. The bacterial cytoskeleton. Annu. Rev. Genet. 44, 365-392 (2010).

3. Wagstaff, J. \& Lowe, J. Prokaryotic cytoskeletons: protein filaments organizing small cells. Nat. Rev. Microbiol. 16, 187-201 (2018).

4. Govindarajan, S. \& Amster-Choder, O. Where are things inside a bacterial cell? Curr. Opin. Microbiol. 33, 83-90 (2016).

5. Ausmees, N., Kuhn, J. R. \& Jacobs-Wagner, C. The bacterial cytoskeleton: an intermediate filament-like function in cell shape. Cell 115, 705-713 (2003).

6. Bartlett, T. M. et al. A periplasmic polymer curves Vibrio cholerae and promotes pathogenesis. Cell 168, 172-185 (2017).

7. Altinoglu, I., Merrifield, C. J. \& Yamaichi, Y. Single molecule super-resolution imaging of bacterial cell pole proteins with high-throughput quantitative analysis pipeline. Sci. Rep. 9, 6680 (2019).

8. Peschek, N., Hoyos, M., Herzog, R., Forstner, K. U. \& Papenfort, K. A conserved RNA seed-pairing domain directs small RNA-mediated stress resistance in enterobacteria. EMBO J. 38, e101650 (2019).

9. Herzog, R., Peschek, N., Frohlich, K. S., Schumacher, K. \& Papenfort, K. Three autoinducer molecules act in concert to control virulence gene expression in Vibrio cholerae. Nucleic Acids Res. 47, 3171-3183 (2019).

10. Davies, B. W., Bogard, R. W., Young, T. S. \& Mekalanos, J. J. Coordinated regulation of accessory genetic elements produces cyclic di-nucleotides for $V$. cholerae virulence. Cell 149, 358-370 (2012).

11. Bradley, E. S., Bodi, K., Ismail, A. M. \& Camilli, A. A genome-wide approach to discovery of small RNAs involved in regulation of virulence in Vibrio cholerae. PLoS Pathog. 7, e1002126 (2011).

12. Kavita, K., de Mets, F. \& Gottesman, S. New aspects of RNA-based regulation by Hfq and its partner sRNAs. Curr. Opin. Microbiol. 42, 53-61 (2018).

13. Gorski, S. A., Vogel, J. \& Doudna, J. A. RNA-based recognition and targeting: sowing the seeds of specificity. Nat. Rev. Mol. Cell Biol. 18, 215-228 (2017).

14. Hör, J., Gorski, S. A. \& Vogel, J. Bacterial RNA biology on a genome scale. Mol. Cell 70, 785-799 (2018).

15. Waters, L. S. \& Storz, G. Regulatory RNAs in bacteria. Cell 136, 615-628 (2009).

16. Wagner, E. G. H. \& Romby, P. Small RNAs in bacteria and archaea: who they are, what they do, and how they do it. Adv. Genet. 90, 133-208 (2015).

17. Cheng, A. T., Ottemann, K. M. \& Yildiz, F. H. Vibrio cholerae response regulator $\mathrm{VxrB}$ controls colonization and regulates the type VI secretion system. PLoS Pathog. 11, e1004933 (2015).

18. Dörr, T. et al. A cell wall damage response mediated by a sensor kinase/ response regulator pair enables beta-lactam tolerance. Proc. Natl Acad. Sci. USA 113, 404-409 (2016).

19. Fong, J. C. et al. Structural dynamics of RbmA governs plasticity of Vibrio cholerae biofilms. Elife 6, e26163 (2017).

20. Papenfort, K., Forstner, K. U., Cong, J. P., Sharma, C. M. \& Bassler, B. L. Differential RNA-seq of Vibrio cholerae identifies the VqmR small RNA as a regulator of biofilm formation. Proc. Natl Acad. Sci. USA 112, E766-E775 (2015).

21. Papenfort, K., Bouvier, M., Mika, F., Sharma, C. M. \& Vogel, J. Evidence for an autonomous $5^{\prime}$ target recognition domain in an Hfq-associated small RNA. Proc. Natl Acad. Sci. USA 107, 20435-20440 (2010).

22. Shin, J. -H. et al. A multifaceted cellular damage repair and prevention pathway promotes high level tolerance to $\beta$-lactam antibiotics. bioRxiv 777375 (2019).

23. Teschler, J. K. et al. Living in the matrix: assembly and control of Vibrio cholerae biofilms. Nat. Rev. Microbiol. 13, 255-268 (2015).

24. Drescher, K. et al. Architectural transitions in Vibrio cholerae biofilms at single-cell resolution. Proc. Natl Acad. Sci. USA 113, E2066-E2072 (2016).

25. Yan, J., Sharo, A. G., Stone, H. A., Wingreen, N. S. \& Bassler, B. L. Vibrio cholerae biofilm growth program and architecture revealed by single-cell live imaging. Proc. Natl Acad. Sci. USA 113, E5337-E5343 (2016).

26. Corcoran, C. P. et al. Superfolder GFP reporters validate diverse new mRNA targets of the classic porin regulator, MicF RNA. Mol. Microbiol. 84, 428-445 (2012).
27. Rehmsmeier, M., Steffen, P., Hochsmann, M. \& Giegerich, R. Fast and effective prediction of microRNA/target duplexes. RNA 10, 1507-1517 (2004)

28. Gitai, Z., Dye, N. A., Reisenauer, A., Wachi, M. \& Shapiro, L. MreB actinmediated segregation of a specific region of a bacterial chromosome. Cell 120, 329-341 (2005)

29. Hartmann, R. et al. Emergence of three-dimensional order and structure in growing biofilms. Nat. Phys. 15, 251-256 (2019).

30. Svenningsen, S. L. Small RNA-based regulation of bacterial quorum sensing and biofilm formation. Microbiol. Spectr. 6, 283-304 (2018).

31. Papenfort, K. et al. A Vibrio cholerae autoinducer-receptor pair that controls biofilm formation. Nat. Chem. Biol. 13, 551 (2017).

32. Casper-Lindley, C. \& Yildiz, F. H. VpsT is a transcriptional regulator required for expression of vps biosynthesis genes and the development of rugose colonial morphology in Vibrio cholerae O1 El Tor. J. Bacteriol. 186, 1574-1578 (2004).

33. Teschler, J. K., Cheng, A. T. \& Yildiz, F. H. The two-component signal transduction system VxrAB positively regulates Vibrio cholerae biofilm formation. J. Bacteriol. 199, e00139 (2017).

34. Nitzan, M., Rehani, R. \& Margalit, H. Integration of bacterial small RNAs in regulatory networks. Annu. Rev. Biophys. 46, 131-148 (2017).

35. Alon, U. Network motifs: theory and experimental approaches. Nat. Rev. Genet. 8, 450-461 (2007).

36. Osella, M., Bosia, C., Cora, D. \& Caselle, M. The role of incoherent microRNA-mediated feedforward loops in noise buffering. PLoS Comput. Biol. 7, e1001101 (2011).

37. Li, L. et al. Sensor histidine kinase is a beta-lactam receptor and induces resistance to beta-lactam antibiotics. Proc. Natl Acad. Sci. USA 113 , 1648-1653 (2016)

38. Typas, A., Banzhaf, M., Gross, C. A. \& Vollmer, W. From the regulation of peptidoglycan synthesis to bacterial growth and morphology. Nat. Rev. Microbiol. 10, 123-136 (2011).

39. Mitchell, A. M. \& Silhavy, T. J. Envelope stress responses: balancing damage repair and toxicity. Nat. Rev. Microbiol. 17, 417-428 (2019).

40. Papenfort, K., Espinosa, E., Casadesus, J. \& Vogel, J. Small RNA-based feedforward loop with AND-gate logic regulates extrachromosomal DNA transfer in Salmonella. Proc. Natl Acad. Sci. USA 112, E4772-E4781 (2015).

41. Gogol, E. B., Rhodius, V. A., Papenfort, K., Vogel, J. \& Gross, C. A. Small RNAs endow a transcriptional activator with essential repressor functions for single-tier control of a global stress regulon. Proc. Natl Acad. Sci. USA 108, 12875-12880 (2011)

42. Thompson, K. M., Rhodius, V. A. \& Gottesman, S. SigmaE regulates and is regulated by a small RNA in Escherichia coli. J. Bacteriol. 189, 4243-4256 (2007).

43. Cabeen, M. T. et al. Bacterial cell curvature through mechanical control of cell growth. EMBO J. 28, 1208-1219 (2009).

44. Kuru, E. et al. In Situ probing of newly synthesized peptidoglycan in live bacteria with fluorescent D-amino acids. Angew. Chem. Int. Ed. Engl. 51, 12519-12523 (2012)

45. Gumpenberger, T. et al. Nucleoside uptake in Vibrio cholerae and its role in the transition fitness from host to environment. Mol. Microbiol. 99, 470-483 (2016).

46. Davies, B. W., Bogard, R. W. \& Mekalanos, J. J. Mapping the regulon of Vibrio cholerae ferric uptake regulator expands its known network of gene regulation. Proc. Natl Acad. Sci. USA 108, 12467-12472 (2011).

47. Boin, M. A., Austin, M. J. \& Häse, C. C. Chemotaxis in Vibrio cholerae. FEMS Microbiol. Lett. 239, 1-8 (2004).

48. Heidelberg, J. F. et al. DNA sequence of both chromosomes of the cholera pathogen Vibrio cholerae. Nature 406, 477-483 (2000).

49. Lim, B., Beyhan, S., Meir, J. \& Yildiz, F. H. Cyclic-diGMP signal transduction systems in Vibrio cholerae: modulation of rugosity and biofilm formation. Mol. Microbiol. 60, 331-348 (2006)

50. Minato, Y. et al. Roles of the sodium-translocating NADH:quinone oxidoreductase (Na+-NQR) on vibrio cholerae metabolism, motility and osmotic stress resistance. PLoS ONE 9, e97083 (2014).

51. Hoyos, M., Huber, M., Forstner, K. U. \& Papenfort, K. Gene autoregulation by 3' UTR-derived bacterial small RNAs. Elife 9, e58836 (2020).

52. Schindelin, J. et al. Fiji: an open-source platform for biological-image analysis. Nat. Methods 9, 676-682 (2012)

53. Ducret, A., Quardokus, E. M. \& Brun, Y. V. MicrobeJ, a tool for high throughput bacterial cell detection and quantitative analysis. Nat. Microbiol 1, 16077 (2016).

54. Singh, P. K. et al. Vibrio cholerae combines individual and collective sensing to trigger biofilm dispersal. Curr. Biol. 27, 3359-3366 e7 (2017).

55. Hartmann, R. et al. BiofilmQ, a software tool for quantitative image analysis of microbial biofilm communities. BioRxiv 735423 (2019).

56. Ayachit, U. The ParaView Guide: A Parallel Visualization Application (Kitware, Inc., New York, 2015).

57. Huber, M., Frohlich, K. S., Radmer, J. \& Papenfort, K. Switching fatty acid metabolism by an RNA-controlled feed forward loop. Proc. Natl Acad. Sci. USA 117, 8044-8054 (2020). 
58. Corpet, F. Multiple sequence alignment with hierarchical clustering. Nucleic Acids Res. 16, 10881-10890 (1988)

59. Ashburner, M. et al. Gene ontology: tool for the unification of biology. The Gene Ontology Consortium. Nat. Genet. 25, 25-29 (2000).

\section{Acknowledgements}

We thank Helmut Blum for help with the RNA sequencing experiments and Andreas Starick, Hannah Jeckel, and Konstantin Neuhaus for excellent technical support. We thank Gisela Storz, Zemer Gitai, and Jörg Vogel for comments on the manuscript and all members of the Papenfort lab for insightful discussions and suggestions. This work was supported by the DFG (TRR174/P5 and P15 and EXC2051- ID390713860), the Human Frontier Science Program (CDA00024/2016-C), The Vallee Foundation, and the European Research Council (StG-758212 and StG-716734) and the Max Planck Society. RH acknowledges support by the Joachim Herz Foundation.

\section{Author contributions}

N.P., R.H., M.S., K.S.F., P.K.S., M.B., K.D., and K.P. designed the experiments; N.P., R.H., P.K.S., M.S., L.S., and K.S.F. performed the experiments; N.P., R.H., P.K.S., M.S., K.S.F., F.M., K.D., and K. P. analyzed data; and K.P. wrote the manuscript with the help of all authors.

\section{Funding}

Open Access funding enabled and organized by Projekt DEAL.

\section{Competing interests}

The authors declare no competing interests.

\section{Additional information}

Supplementary information is available for this paper at https://doi.org/10.1038/s41467020-19890-8.

Correspondence and requests for materials should be addressed to K.P.

Peer review information Nature Communications thanks the anonymous reviewers for their contribution to the peer review of this work.

Reprints and permission information is available at http://www.nature.com/reprints

Publisher's note Springer Nature remains neutral with regard to jurisdictional claims in published maps and institutional affiliations.

(c) (i) Open Access This article is licensed under a Creative Commons Attribution 4.0 International License, which permits use, sharing adaptation, distribution and reproduction in any medium or format, as long as you give appropriate credit to the original author(s) and the source, provide a link to the Creative Commons license, and indicate if changes were made. The images or other third party material in this article are included in the article's Creative Commons license, unless indicated otherwise in a credit line to the material. If material is not included in the article's Creative Commons license and your intended use is not permitted by statutory regulation or exceeds the permitted use, you will need to obtain permission directly from the copyright holder. To view a copy of this license, visit http://creativecommons.org/ licenses/by/4.0\%

(C) The Author(s) 2020 